lessly to the grave in pain and distress. What the risks are in non-malignant cases where gastro-enterostomy is done it is impossible to state as yet in figures. In this case the risk was probably greater than had the operation been done before the extreme emaciation had been reached with which the patient came into hospital. But, at all events, they appear not to be greater than those accepted daily in other operations for conditions by no means so desperate. In this connection I may be allowed to say that among a considerable number of gastro-enterostomies which I have done I have never seen any trace of that kinking of the attached bowel which appears to have been so fatal in many cases. This may be due to the fact that for some years the method employed has been the retrocolic, which appears to me to have many advantages over the original anterior method. Again, I see no advantage in the use of Murphy's button in these cases, although I have used it in earlier cases with success and without any untoward occurrences. It may save a little time when we are not practised in these operations but not much now. This operation was completed in forty-four minutes.

\section{REMARKS ON A CASE OF RETROPERITONEAL CYST.}

By JOHN WARD COUSINS, F.R.C.S., M.D.LoND., Senior Surgeon to the Royal Portsmouth Hospital and South Hants Eye
and Ear Infirmary.

THE pathology of retroperitoneal cysts is still incomplete. In some cases these growths have been not only developed in the immediate neighbourhood of the pancreas, but they have been found to contain within their structure pancreatic glandular tissue. Cases have been recorded in which cystic tumours have been intimately connected with the whole gland or attached to the pancreas by a well-defined pedicle. The gland has been known to atrophy from the direct pressure of the growth, or from the invasion of its structure by the expansion and development of the cyst itself. Cysts have been found undergoing purulent degeneration, and their contents have been mixed with blood clot or mingled with the traces of bygone hæmorrhage. As regards the presence in cystic formations of distinct pancreatic secretion, I believe it has been discovered only in small and recent growths.

Retroperitoneal cysts have been attributed to various causes. Some have regarded them as the outcome of the organisation of plastic exudation around blood clot; others have explained their origin in the localised changes excited by inflammation of the pancreas itself. A cystic formation may commence from a small rupture of the gland followed by parenchymatous hæmorrhage, and the spaces thus produced are soon filled with blood and pancreatic secretion. It is probable that pancreatic cysts are generally the result of traumatism, or are caused by obstruction of the excretory ducts and retained secretion. Besides, however, internal lesions within the pancreas itself, calculi have been found lodged in the duct of Wirsung; the ducts of the gland have been compressed by the development of new growths, and pancreatic retention has been known to follow plugging of the ductus choledochus with a gall stone near its orifice.

Sometimes retroperitoneal cysts are associated with malignant tumours. Two years since a woman in advanced life came under my care with a large abdominal cyst. She had suffered a great deal of pain, while marked cachexia and emaciation were present. The swelling was central and prominent, and was surrounded by intestinal resonance. When the abdomen was opened the cyst could be traced to a deep and broad attachment. It presented below the transverse colon, and its surface was covered with distended vessels. The fluid contents were septic, thick, yellow, and offensive. The cyst was fixed to the lower end of the wound and drained. She found great relief from the treatment, and left the hospital, but I have not been able to trace her since.

In the following case the pancreatic origin of the cyst was undetermined, and the only evidence in favour of such a conclusion was the position of the tumour and its deep attachment discovered at the time of the operation.
H. P., a married woman, and the mother of two children, came under my care in December, 1897 .

History.-After the birth of the youngest child she had two miscarriages. On her admission to the Royal Portsmouth Hospital she stated that the abdomen had been increasing in size for many months, and she thought that she was again pregnant. The swelling commenced near the umbilicus, and she had suffered no pain or any disturbance of her health. On examination the abdomen exhibited a well-marked swelling extending from 3 inches above the umbilicus to the pubes. The long axis ran downwards obliquely to the left, and a distinct sulcus was situated over the umbilicus. The walls of the abdomen were elastic and moved freely on the tumour, and its outline was clearly discernible. Fluctuation was very distinct in all directions. The circumference of the abdomen at the umbilicus measured 37 inches. The pelvic organs were healthy, and the uterus normal in size and freely movable. Resonance on percus sion was well marked in the left flank and over both inguinal regions. Operation.-Laparotomy was performed on December 27 th, 1897. The surfaces of the cyst when exposed were found to be continuous with the inferior layer of the mesocolon, the descending colon presented on the eft, and the small intestines overlapped it below. The tumour extended backwards towards the centre of the abdomen, and the vessels in every direction were much distended. Six pints of a clear and slightly yellowish fluid were removed by aspiration, and the operation was completed by dring the cyst to the lower angle of the wound and inserting a glass chare characters. Clear, alkaline, very slightly coloured, specific gravity ro2o, After-History.-During the following two weeks a small quantity of the After-History.-During the following two. Weeks a smanl quantity of the interior of of fluid was drawn off daily with a glass syringe, and the interior of the cyst washed out with sulphurous acid lotion. The wound rapidly healed and the patient left the hospital on January ${ }^{27}$ th, ${ }^{1898}$. In the month of November she was confined with her third child. I saw her again early last year, she was then in good health. The abdominal detected at the lower angle.

The physical examination of the abdomen clearly indicated the presence of a large cystoma, but its origin and relations were uncertain. The central swelling, the resonance in the inguinal regions, and the condition of the pelvic organs contraindicated the existence of an ovarian or broad ligament cyst. When the abdomen was opened the surface of the tumour appeared to be overlapped in all directions by the surrounding organs, and the mesenteric vessels could be seer stretched over its exterior. It was partially movable from side to side, with its base firmly and deeply fixed in the abdominal cavity. After the evacuation of the fluid contents it was very easy to make out its lumbar attachment and its retroperitoneal relations. When the cyst was drawn forwards into the wound, it carried with it the whole mass of intestines both large and small. I did not introduce my hand into the interior as the empty sac contracted firmly, and as it appeared probable that the manipulation would be attended with some risk.

A cyst may be developed behind the stomach, and behind or within the peritoneal sac, and may ultimately reach the surface by displacing that organ upwards and pushing forwards the lesser omentum. In this position it will protrude itself above the transverse colon. In my case the tumour was found in contact with the abdominal wall just below the umbilicus, the great omentum was puckered up to the right, the transverse colon partly covered the upper part, and the descending colon overlapped it on the left.

The diagnosis of small retroperitoneal cysts is often impossible, as they are covered up completely by the abdominal organs; but, when a tumour lias reached a considerable size, a fluctuating swelling can be detected in the centre of the cavity. In many recorded cases of genuine pancreatic cysts the patients have suffered pain and marked debility. Sometimes diabetic symptoms have been observed, and also the presence of fatty matter in the stools. In a few cases jaundice has occurred from pressure on the common bile duct, and also sudden death caused by the rupture of a large cyst and internal hæmorrhage.

In my case the patient suffered from no pain and very little inconvenience, and for many months she considered herself pregnant. There was at no time any reflex cardiac disturbance or dyspeptic trouble from pressure on the nerves of the solar plexus. Her health continued good, and she carried on without interruption her household duties.

In all cases of doubt and difficulty it is my practice to máke an exploratory incision, for in no other way can the relations and character of a disorder be determined. Paracentesis is a very unsatisfactory proceeding, and practically reveals nothing, and is certainly not always free from risk. The escape of some kinds of cystic fluid into the peritoneal cavity may excite serious symptoms, and the accidental puncture of a dilated vessel may be followed by fatal hæmor- 
rhage. In my.case no attempt was made to enucleate the cyst. It was simply incised and drained, and this treatment in a large number of similar cases has proved very successful. In $1897 \mathrm{Mr}$. Alban Doran stated that he had collected seventy cases treated in this way, and that of this number sixty-five had recovered.

Many retroperitoneal cysts with deep and central connections have been successfully removed, but I do not think there are many cases in which this operation can be undertaken without considerable danger to life. In any case, however, where very little enucleation is required, and a welldefined pedicle is present, which can be transfixed with a needle, leaving only a small cut surface after the separation of the tumour, the operation may be undertaken with a good prospect of success. Sir Spencer Wells recorded a case, diagnosed as an ovarian tumour, which was operated on by Bozeman. Twenty pints of fluid were drawn off, the pedicle tied, and the cyst cut away. But there are other cases in which the serious connections of the tumour prevented the completion of the operation. The difficulty of extirpation arises from the deep and almost inaccessible position of the base of the growth, and the important nervous and vascular structures with which it is connected. Portions of the pancreas have sometimes been excised with the cyst and the splenic artery ligatured. These operations are occasionally followed by very troublesome fistulæ.

\section{BIBLIOGRAPHY.}

System of Practical Medicine, Loomis and Thompson, vol. iii. Diagnosis and Treatment of Abdominal Tumours, Sir Spencer Wells, p. 206 . Friedreich, Practice of Medicine, Ziemssen, vol. viii. Annandale, BRITISH MEDICAL JOURNAL, vol. i, 1889, p. 1296. Savill, Lancet, vol. i, 1891, p. 666. Alban Doran, BRITISH Medical JouRNaL, vol. ii, 1897, p. 1779 .

\section{STRANGULATED FEMORAL HERNIA : SUCCESSFUL PRIMARY RESECTION OF THE DAMAGED GUT.}

BY HENRY BETHAM ROBINSON, M.S.LoND., F.R.C.S., Assistant Surgeon to, Surgeon in charge of the Throat Department, and Lecturer on Anatomy at St. Thonas's Hospital; Surgeon to the
East London Hospital for Children, Shadwell.

UP to the present time it may be considered a still debatable point as to the best course to pursue when the state of the bowel indicates that its recovery is beyond hope. In many cases our course is decided by the condition of the patient ; there is no alternative to leaving the diseased gut in situ and allowing an artificial anus to form, this lesion to be repaired later by some method of secondary operation. If the patient's condition is good, a primary operation may be done with a very good prospect of success, and certainly a great saving of time. As to how the resected bowel should be joined, whether by mechanical aids or by simple suturing, this depends on the opinion of the surgeon. Mr. Jonathan Hutchinson junior's statistical paper on the subject in vol. xxxii Transactions of the Clinical Society shows a marked superiority of suturing, 46 per cent. of recoveries against 7 per cent. for those where Murphy's button was used, which 46 per cent. might have been improved by the inclusion of another successful case of my own.1 These figures, however, point forcibly to the conclusion already held by most surgeons that simple suturing, if expeditionsly carried out, is more certain of accurate apposition, and has the gieat advantage of removing all anxiety as to the ultimate fate of the button.

From the technique employed in the following case three points may be drawn attention to: (I) The accurate apposition of the mesenteric border of the cut ends was attained by the stitch similarly used by Maunsell in his method of resection. (2) The adoption of a continuous Lembert's suture whereby the sutured edges are more evenly supported and much time is saved. This method I adopted in my other case. (3) The resection in situ, instead of making an abdominal incision, withdrawing the damaged gut and running the risk of fouling the peritoneal cavity. Should any difficulty be met with in replacing the bowel, the opening can be enlarged and then resutured without causing any weakening in the lower part of the abdominal wall.
S. S., 45, married, was admitted into St. Thomas's Hospital under my care on June 6th, 1899, with a strangulated iemoral hernia.

History.
The hernia, which ? was on the riglt side, was first noticed some seven weeks before, coming on after a severe attack of vomiting (? cause). She was fitted with a truss, and suffered no further inconvenience until June 4hl, when she was suddenly taken ill in church with pain and vomiting.
The hernia was found to be irreducible. The constant vomiting with severe abdominal pain continued for forty-eight lours, when she sought admission into the hospital.

Condition on Admission. She was found to have a small tense right femoral hernia without any
impulse, and with some cedema of skin over it. Her pulse was 84 , respi-

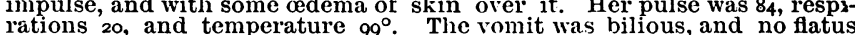

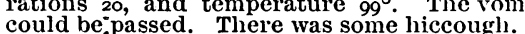

\section{Operation.}

Immediate operation was advised and procecded with. After opening the hernial sac in the usual manner and setting free some turbid fluid, it was found that only a small knuckle of gut occupied the sac. This gut was very black, thickened, and covered with lympl in patches, and after relieving the constriction and drawing down the gut, it was seen that the upper end was ulcerated through down to the serous layer, and the lower-
point of constriction was also thinned. From the state of the interpoint of constriction was also thinned. From the state of the interit was determined to do immediate resection rather than allow an artificial anus to be formed.

The resection was able to be done in situ. Makins's clamps were applied above and below the lines of constriction, and three inches of bowel were removed with scissors, with the corresponding portion of mesentery. All bleeding points were ligatured with fine silk and the cut mesenteric edges united. A fixation stitch, as in Maunsell's method, was then passed between the mesenteric edges of the cut ends of the bowel and they were brought close together, with the serous layers apposed and the mucous edges inverted. A continous catgut stitch then brough the mucous edges together without any constriction, and a continuous Lembert's suture of fine silkworm gut was then passed through the seromuscular coat. With slight enlargement of the wound upwards inte Poupart's ligament the gut was; easily replaced in the abdomen, after sponging it with normal saline solution. The sac was separated, ligatured, and removed. The pectineal fascia was dissected up, and stitched over the femoral opening to Poupart's ligament, which was itself resutured up where it had been divided. After suturing the wound with horsehair it was dressed with cyanide gauze. The whole operation took about forty-five minutes, and the patient's condition was most satisfactory her pulse maintaining its former rate of 84 per minute.

\section{After-History.}

On the evening of the operation she was given a simple enema, and then one of a pint of normal saline solution. Temperature $99.4^{\circ}$. Put on rectal feeding.

June 7 th. Condition very comfortable. Temperature $98.8^{\circ}$; pulse 80 Allowed a teaspoonful of warm water by mouth every hour. In the evening temperature $100.2^{\circ}$, respirations 24 , pulse 84

June 8 th. Flatus passed during the niglit. Temperature $99^{\circ}$, pulse 60 .

Given teaspoonfuls of egg albumen and brandy.
June gtli. Temperature $99^{\circ}$. Milk and water ordered; given simple enema.

June roth. Temperature normal, and continued so ; calvesfoot jelly.

June irth. Raw meat juice given

June rath. Very comfortable. Taking now beei-tea Oj, milk Ojss, and eggs.

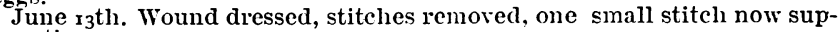
purating.

Jume $5^{\text {th. }}$ Bowels opened naturally; given milk puddings.

ind wound healed.

June 2 ist. Allowed to get up.

June 27 th. Left the hospital quite well.

REFERENCE.

1 Lancet, 1897, vol. i, p. 1614.

\section{SOME REMARKS ON THE RADICAL CURE OF HERNIA ; BASED ON 190 CASES OF OPERA- TION FOR THE CURE OF OBLIQUE INGUINAL HERNIA.*}

By A. R. ANDERSON, F.R.C.S.,

Surgeon to the General Hospital, Nottingham.

I HAve chosen this as the subject of my address for two reasons-first, because it is one of which I have had a fair amount of experience, and secondly, because there is perhaps no other operation in surgery about which so much difference of opinion exists, and none about which more has been said or written during recent times. Early in 1899 I read before the Nottingham Medico-Chirurgical Society a paper detailing I 7 cases of operation for radical cure, and since then $I$ have operated on 73 cases, bringing the total number of operations

* An abstract of the Presidential Address to the Midland Branch of the British Medical Association. 